\title{
Rapid Partial Repigmentation of Vitiligo in a Young Female Adult with a Gluten-Free Diet
}

\author{
Birgit N. Khandalavala Maya C. Nirmalraj \\ Department of Family Medicine, University of Nebraska Medical Center, Omaha, Nebr., \\ USA
}

\section{Key Words}

Vitiligo · Autoimmune diseases - Dietary therapy

\begin{abstract}
Vitiligo is a common pigmentary skin condition with a profound impact. Despite a number of therapeutic modalities, few have been demonstrated to result in significant repigmentation within a brief period of time. Reported dietary interventions are sparse. Following exclusion of gluten in the diet, early and extensive repigmentation of facial lesions were noted in a young female adult of Asian ethnicity with acrofacial vitiligo. The majority of the benefits occurred within the first month and stabilized at 4 months. Previous topical and phototherapy had not been found to be effective. The patient was maintained on the previously prescribed dapsone therapy. Dietary elimination can potentially be a disease-modifying intervention for vitiligo and should be considered even in patients without concomitant celiac disease.

(c) 2014 S. Karger AG, Basel
\end{abstract}

\section{Case Report}

A 22-year-old female of South Indian ethnicity with a 3-year history of acrofacial vitiligo presented for consultation in July 2013. She had been born, raised and lived exclusively in the United States. There were no previous medical concerns or history of colitis until asymptomatic areas of depigmentation appeared in June 2010 on the lateral side of the left eye. A few weeks later, symmetrical lesions were detected around the right side of the eye. Depigmentation progressed over a period of 6 months, both superiorly and inferiorly over both eyes, to converge at the medial aspect of the orbit. During this time, additional facial lesions were noted on the nose. Nine months later, lesions started appearing over the acral 
aspects of both upper and lower limbs and continued to progress. Review of systems was pertinent for the absence of any gastrointestinal or systemic symptoms. There was no personal or family history of celiac disease or of any other autoimmune disorders. The maternal grandmother and father had diabetes. Physical examination revealed extensive crenated depigmented lesions over the face and extremities with sparing of the torso (fig. 1). There were no signs suggestive of other concomitant autoimmune disorders. Biochemical evaluation, including a complete metabolic profile, sedimentation rate, blood count and thyroidstimulating hormone, did not indicate any thyroid or metabolic abnormalities.

Topical therapy had been initiated within the first year with tacrolimus ointment, and subsequently calcipotriene, with no response, following a diagnosis of vitiligo by a local dermatologist. Topical steroids had been equally ineffective. Dapsone had been initiated at $100 \mathrm{mg}$ three times a week with simultaneous phototherapy, after consulting a dermatologist with extensive expertise in vitiligo in India. The light therapy had been discontinued due to inconvenience and lack of response. Based on anecdotal improvement of vitiligo with elimination of gluten from other patients and the lack of any significant impact of conventional therapies, we advised her to try complete elimination of gluten from her regular diet to determine whether this would result in any potential changes. She was maintained on oral dapsone. Within 1 month, profound and significant repigmentation was evident (fig. 2). Of note, the areas of repigmentation were distinctly darker than the native skin, which normalized over the subsequent month. Improvement continued for the next 3 months and maximal improvement was seen by 3 months, with no further repigmentation (fig. 3). None of the other lesions showed similar changes or progress of the disease. The elimination diet was well tolerated, though restrictive, and compliance was not difficult to maintain once benefits were evident. Long-term follow-up is planned.

\section{Discussion}

Vitiligo is a challenging chronic disease with a profound impact on the life of patients, with all options having limited success [1]. The goal of treatment is repigmentation, which occurs by activation and migration of melanocytes from their reservoirs that are found in hair follicles [1]. Facial lesions are known to respond best [1], as demonstrated in our case. Topical corticosteroids and ultraviolet light therapy used alone or in combination [2] have been noted to have limited success, however were not effective in this patient. The use of dapsone in patients with vitiligo has been described [3,4]. This regimen was noted to stabilize the progression of the disease; however, no repigmentation manifested over the next 2 years. Dapsone has a well-validated history of use in various dermatological conditions with few adverse effects [5], and this was noted in our patient's case. A synergistic relationship with dapsone and a gluten-free diet could potentially be inferred from a similar recommendation to prevent relapses in dermatitis herpetiformis [6].

There are minimal reports in the published literature of dietary interventions for vitiligo as a treatment modality. In celiac disease and for associated dermatitis herpetiformis, the elimination of gluten has been associated with significant improvement [7]. Since celiac disease is commonly associated with vitiligo, in patients who have both conditions, eliminating gluten will be particularly advantageous. This was evident in the case report of a 9-year-old child with celiac disease with extensive repigmentation of the abdominal lesions over a period of 6 years [8]. Gluten-free diets are becoming more widely available and are easier to accommodate in today's lifestyle, with most of the indications being in patients with known celiac disease. 
Repigmentation typically is a slow process which usually requires at least 6-12 months of treatment with conventional modalities [1]. Rapid initiation of repigmentation has been reported from Europe with pseudocatalase therapy, with results evident as early as 10 days [9]. Using a novel formulation, a recent pilot study showed rapid results of repigmentation within an average of 11.25 weeks of treatment [10]. Our patient was surprised to detect visible changes within the first 2 weeks that progressed quickly within the first month and appeared to slow down over the next 3 months, with no further changes after 4 months. This simple modification was found to have had the biggest impact of all the medical therapies that had been undertaken for the previous 3 years, involving a variety of medications and phototherapy. No extensive evaluation and testing were undertaken, with minimal cost incurred for the patient. Though there could be a number of explanations for the regression, including medication effect and spontaneous remission, the patient had been on a prior stable unchanged regimen for 2 years, and the abrupt changes following drastic changes in diet would certainly implicate more of a causal etiology. Re-challenging with gluten would be more conclusive and has been recommended, however it is not an option that the patient is willing to pursue.

To the best of our knowledge, after a thorough search of the published literature that included PubMed (Medline), Embase, Cochrane and Scopus, this is the first documented case of rapid repigmentation of acrofacial vitiligo utilizing elimination of gluten in an adult patient without concomitant celiac disease. This novel approach certainly needs to be further explored. It would appear from both cases mentioned here that eliminating gluten at an early stage of the disease could have a potentially encouraging and disease-modifying effect.

\section{Patient's Perspective}

Seeing the first few areas of white patches during my first year of college was psychologically and socially devastating. My personality changed, with previously unknown overwhelming social apprehension and loss of self-esteem. My vitiligo is the first thing I think of now, and I long for a day where I feel entirely comfortable in my skin. Lack of awareness of the disease is the biggest issue, as I believe if more people were educated, patients would not feel so insecure, anxious and afraid. Going through a variety of treatments with little success, the return of color to my facial lesions has been motivating and the diet easy to follow, and I strongly recommend that dietary manipulations be considered more often. My wish is that remission will continue and that others with my disease will be given the opportunity to not only try this simple and effective treatment, but to be offered additional therapies for longterm success.

\section{Disclosure Statement}

No financial support has been received for the work reported. 
Khandalavala and Nirmalraj: Rapid Partial Repigmentation of Vitiligo in a Young Female Adult with a Gluten-Free Diet

\section{References}

1 Habif TP: Clinical Dermatology: A Color Guide to Diagnosis and Therapy, ed 5. Edinburgh, Elsevier, 2010, pp 764-769.

-2 Whitton ME, Pinart M, Batchelor J, Lushey C, Leonardi-Bee J, González U: Interventions for vitiligo. Cochrane Database Syst Rev 2010;1:CD003263.

3 Salafia A: Vitiligo. Successful treatment with dapsone. Chron Dermatol 1995;5:171-188.

4 Salafia A: Vitiligo-Leukoderma: Textbook and Atlas. Dr. Antonio Salafia, 2010.

5 Habif TP: Clinical Dermatology: A Color Guide to Diagnosis and Therapy, ed 5. Edinburgh, Elsevier, 2010, pp 367-369.

6 Celiac Disease Foundation: Dermatitis herpetiformis. Woodland Hills, Celiac Disease Foundation, (C2014. Available at: http://celiac.org/celiac-disease/dermatitis-herpetiformis/.

7 Wozel G, Blasum C: Dapsone in dermatology and beyond. Arch Dermatol Res 2014;306:103-124.

-8 Rodríguez-García C, González-Hernández S, Pérez-Robayna N, Guimerá F, Fagundo E, Sánchez R: Repigmentation of vitiligo lesions in a child with celiac disease after a gluten-free diet. Pediatr Dermatol 2011;28:209-210.

-9 Schallreuter KU, Moore J, Behrens-Williams S, Panske A, Harari M: Rapid initiation of repigmentation in vitiligo with Dead Sea climatotherapy in combination with pseudocatalase (PC-KUS). Int J Dermatol 2002;41:482-487.

10 Goren A, Salafia A, McCoy J, Keene S, Lotti T: Novel topical cream delivers safe and effective sunlight therapy for vitiligo by selectively filtering damaging ultraviolet radiation. Dermatol Ther 2014;27:195-197.

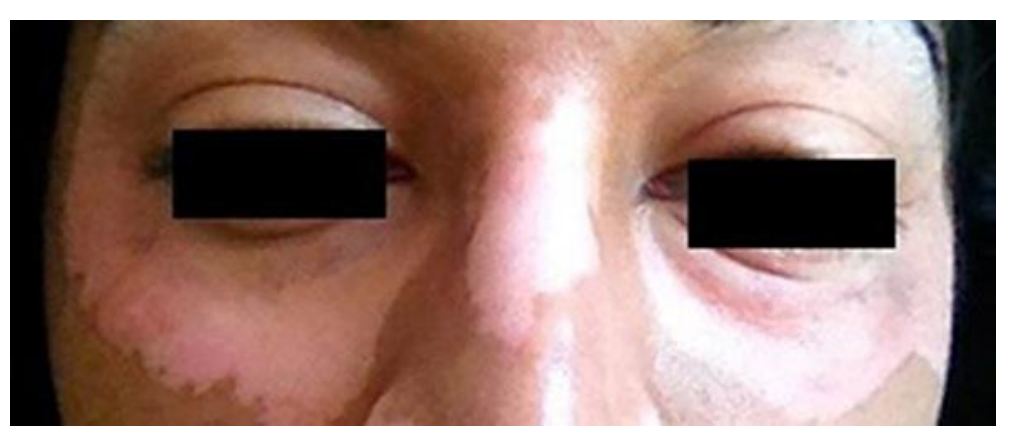

Fig. 1. July 18, 2013.
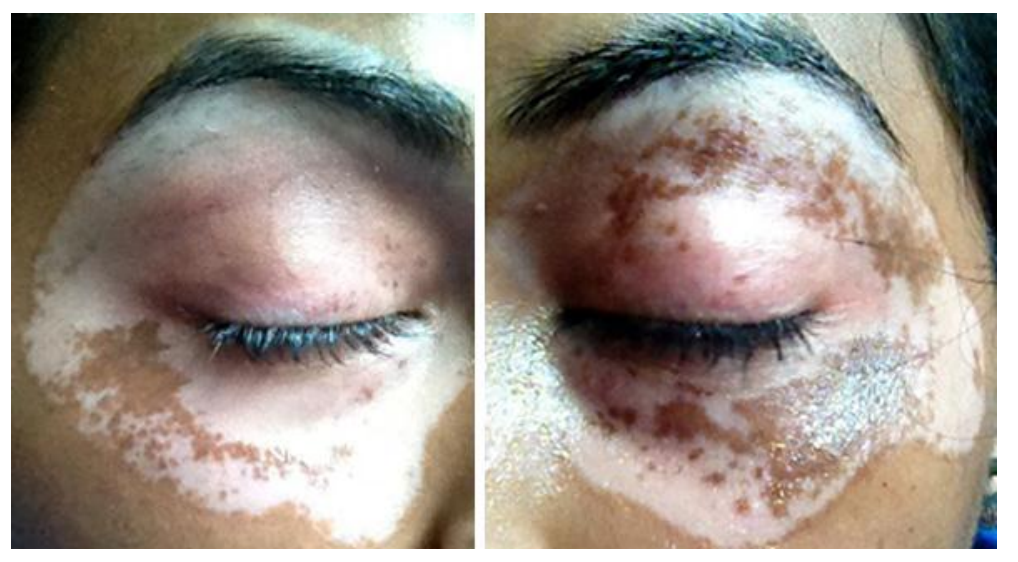

Fig. 2. August 12, 2013. 
Case Reports in
Dermatology

Case Rep Dermatol 2014;6:283-287

DOI: 10.1159/000370303

(c) 2014 S. Karger AG, Basel

Khandalavala and Nirmalraj: Rapid Partial Repigmentation of Vitiligo in a Young Female Adult with a Gluten-Free Diet
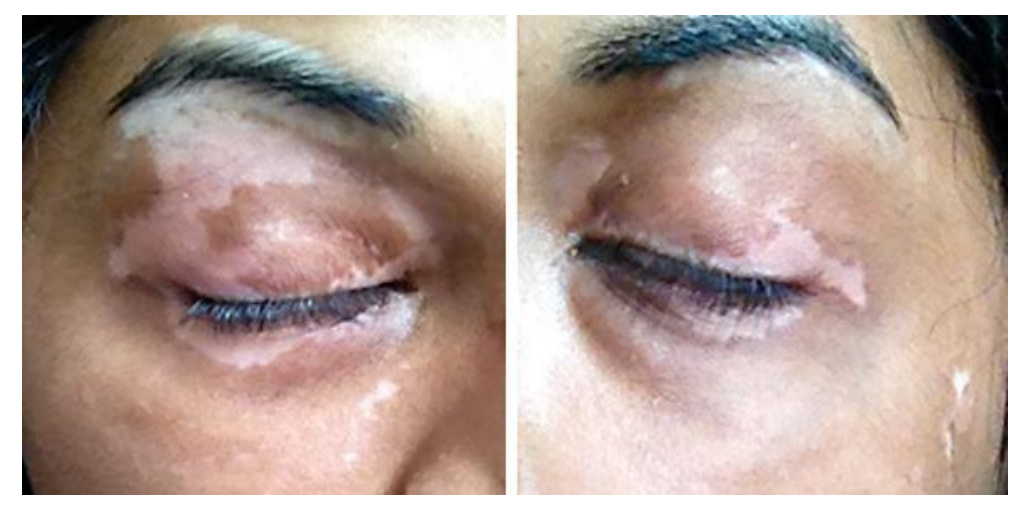

Fig. 3. October 3, 2013. 